\title{
Differential Regulation of Multiple Hepatic Protein Tyrosine Phosphatases in Alloxan Diabetic Rats
}

\author{
Joan M. Boylan,"* David L. Brautigan, " Jane Madden, Thomas Raven, Leland Ellis," and Philip A. Gruppuso** \\ *Section of Biochemistry, Division of Biology and Medicine, Brown University, Providence, Rhode Island 02912; ${ }^{\ddagger}$ Department of \\ Pediatrics, Rhode Island Hospital, Providence, Rhode Island 02903; 8 Biochemistry Department, Glaxo Group Research Limited, \\ Greenford, Middlesex, UB6 OHE, United Kingdom; and "Howard Hughes Medical Institute and Department of Biochemistry, University \\ of Texas Southwestern Medical Center, Dallas, Texas 75235-9050
}

\begin{abstract}
The involvement of tyrosine phosphorylation in insulin action led us to hypothesize that increased activity of protein tyrosine phosphatases (PTPases) might contribute to insulin resistance in alloxan diabetes in the rat. Hepatic PTPase activity was measured using two artificial substrates phosphorylated on tyrosine: reduced, carboxyamidomethylated, and maleylated lysozyme (P-Tyr-RCML) and myelin basic protein (P-TyrMBP), as well as an autophosphorylated $48-\mathrm{kD}$ insulin receptor tyrosine kinase domain (P-Tyr-IRKD). Rats that were made alloxan diabetic exhibited a significant increase in hepatic membrane (detergent-soluble) PTPase activity measured with $\mathbf{P}-\mathrm{Tyr}-\mathrm{MBP}$, without a change in activity measured with $\mathbf{P}$ Tyr-RCML or the P-Tyr-IRKD. The PTPase active with $\mathbf{P}$ Tyr-MBP behaved as a high molecular weight peak during gel filtration chromatography. Characterization of this enzyme indicated it shared properties with CD45, the prototype for a class of transmembrane, receptor-like PTPases. Our results indicate that alloxan diabetes in the rat is associated with an increase in the activity of a large, membrane-associated PTPase which accounts for only a small proportion of insulin receptor tyrosine dephosphorylation. Nonetheless, increased activity of this PTPase may oppose tyrosine kinase-mediated insulin signal transmission, thus contributing to insulin resistance. (J. Clin. Invest. 1992.90:174-179.) Key words: protein tyrosine phosphatases $\bullet$ diabetes $\bullet$ insulin $\bullet$ hormone receptors
\end{abstract}

\section{Introduction}

The insulin receptor is a tyrosine specific protein kinase that is activated by autophosphorylation after the binding of insulin (reviewed by Rosen [1]). Although the specific events that follow insulin receptor tyrosine kinase activation have not been

\footnotetext{
Address correspondence and reprint requests to Dr. Philip A. Gruppuso, Pediatric Endocrinology and Metabolism, Rhode Island Hospital, 593 Eddy Street, Providence, RI 02903. The present address of Dr. Leland Ellis is Institute of Biosciences and Technology, Texas A \& M University, 2121 Holcombe, Houston, TX 77030.

Received for publication 4 June 1991 and in revised form 26 February 1992.
}

J. Clin. Invest.

(c) The American Society for Clinical Investigation, Inc. 0021-9738/92/07/0174/06. \$2.00

Volume 90, July 1992, 174-179 elucidated, protein tyrosine phosphatases (PTPases) ${ }^{1}$ may be involved in attenuating insulin effects by reversing tyrosine phosphorylation. Increased PTPase activity might lead to resistance to the metabolic and/or anabolic effects of insulin via dephosphorylation of the autophosphorylated receptor kinase or its substrates. Such a hypothesis is supported by recent studies showing that injection of PTPase into oocytes delays the action of insulin on maturation (2).

Available sequence information shows that PTPases fall into two broad categories (reviewed by Fischer et al. [3]): (a) those that have one catalytic domain $\left(50,000 M_{\mathrm{r}}\right)$; and $(b)$ those which have one or two intracellular catalytic domains, a single membrane spanning region, and an external $\mathrm{NH}_{2}$-terminal region $\left(160,000-200,000 M_{r}\right)$. These intracellular versus transmembrane PTPases show different substrate specificity. PTPase 1B from human placenta, a catalytic fragment of an intracellular PTPase, was shown to be specific for phosphotyrosine (Tyr[P]) containing proteins. However, it exhibited a relative lack of selectivity for the protein bearing the $\operatorname{Tyr}(\mathrm{P})$ residue, showing similar specific activities and $K_{\mathrm{m}}$ for several artificial substrates tested; reduced carboxyamidomethylated and maleylated lysozyme (P-Tyr-RCML), reduced carboxyamidomethylated and succinylated bovine serum albumin (PTyr-RCS-BSA), both polyacidic substrates, and myelin basic protein (P-Tyr-MBP), a polybasic substrate (4). In contrast, CD45, a leukocyte transmembrane PTPase, exhibited less than $5 \%$ of the specific activity of PTPase 1B when using P-TyrRCML as substrate (5). However, with P-Tyr-MBP as substrate, CD45 had $\sim 2.5$-fold higher specific activity than PTPase 1B.

We have previously reported the effects of alloxan diabetes on rat hepatic PTPase activity measured using the polyacidic substrates P-Tyr-RCML and P-Tyr-poly[glutamic acid:tyrosine (4:1)] (P-Tyr-polyEY) (6). Our data indicated no changes in PTPase activity in various subcellular fractions; cytosol, membrane (detergent soluble), and cytoskeleton (detergent insoluble, extracted with $0.6 \mathrm{M} \mathrm{KCl}$ ). PTPase measured with these substrates was consistent with assignment of activity to an enzyme or enzymes similar to human placental PTPase 1B (5). Subsequent to these studies we undertook the characteriza-

\footnotetext{
1. Abbreviations used in this paper: IRKD, insulin receptor tyrosine kinase domain; LAR, leukocyte antigen related; PTPases, protein tyrosine phosphatases; P-Tyr-polyEY, poly(glutamic acid: tyrosine [4:1]) phosphorylated on tyrosine; P-Tyr-IRKD, IRKD phosphorylated on IRKD; P-Tyr-MBP, myelin basic protein phosphorylated on tyrosine; P-Tyr-RCML, reduced carboxyamidomethylated and maleylated lysozyme phosphorylated on tyrosine; $\operatorname{Tyr}(\mathrm{P})$, phosphotyrosine.
} 
tion of rat liver PTPases (7) and found several distinct membrane PTPases. PTPases I and II partially purified from rat liver extracts could be separated by poly-L-lysine agarose affinity chromatography. Their characteristics included molecular mass ranging from 40 to $85 \mathrm{kD}$, based on gel filtration chromatography, and a 6- to 10-fold greater activity with P-Tyr-RCML than with P-Tyr-MBP. Also present during poly-L-lysine chromatography of membrane proteins was a major peak of P-TyrMBP PTPase activity that showed little activity with P-TyrRCML. This activity, designated PTPase III, was $\sim 400 \mathrm{kD}$ as estimated by gel filtration chromatography. Because of the substrate specificities of these enzymes, and the exclusive use of polyacidic substrates in our earlier experiments on alloxan diabetes, an effect on the class of transmembrane PTPases with preference for polybasic substrates could not be ruled out. We therefore undertook the present studies to determine the effects of alloxan diabetes on the latter class of hepatic PTPases.

\section{Methods}

Animal studies. Male Sprague-Dawley rats (Charles River Laboratories, Wilmington, MA) weighing 150-175 g were made diabetic by the intravenous administration of alloxan, $65 \mathrm{mg} / \mathrm{kg}$, as described previously (6). This model of diabetes produces hyperglycemia, insulinopenia, and elevated plasma beta-hydroxybutyrate concentrations, although ketonemia is insufficient to produce acidosis (6). A separate group of alloxan diabetic animals was treated with insulin ( $2 \mathrm{U}$ recombinant human insulin [Humulin; Eli Lilly and Co., Indianapolis, IN] i.p. every $12 \mathrm{~h}$ ) beginning $3 \mathrm{~d}$ after alloxan injection for $48 \mathrm{~h}$ before killing (6). As a control for alloxan diabetes, which is associated with weight loss, a group of animals was fasted for $48 \mathrm{~h}$ (ad lib. water intake).

The method of Williams et al. (8) was used to prepare unfractionated membranes from individual fresh livers. The supernatants from membrane preparations were retained for measurement of soluble (cytosolic) activity. Detergent extracts of membrane preparations, referred to as the membrane fraction, were made by diluting 1 vol of the unfractionated membrane suspension $(10 \mathrm{mg}$ protein $/ \mathrm{ml})$ with 1 vol $50 \mathrm{mM}$ Hepes, pH 7.4, 2\% (vol/vol) Triton X-100. After 5 min on ice, insoluble material was collected by centrifugation at $40,000 \mathrm{~g}$ for $20 \mathrm{~min}$ and extracted with 0.5 vol $25 \mathrm{mM}$ Hepes, $\mathrm{pH} 7.4,0.6 \mathrm{M} \mathrm{KCl}$. This second extract, clarified by centrifugation at $40,000 \mathrm{~g}$ for $20 \mathrm{~min}$, is referred to as the cytoskeletal fraction. Protein concentrations were determined by the bicinchoninic acid method (BCA, Pierce Chem. Co., Rockford, IL) using BSA as standard.

PTPase assays. PTPase activity was measured as the release of ${ }^{32} \mathrm{P}_{\mathrm{i}}$ from artificial substrates phosphorylated on tyrosine (9). The present studies used P-Tyr-RCML and P-Tyr-MBP as substrates. Dephosphorylation of the artificial substrates was measured at a final $\operatorname{Tyr}(\mathrm{P})$ concentration of $1 \mu \mathrm{M}$ (specific radioactivity, $\sim 400 \mathrm{cpm} / \mathrm{pmol}$ ) in the presence of $1 \mathrm{mM}$ ethylenediaminetetraacetate and $15 \mathrm{mM} 2$-mercaptoethanol. Dephosphorylation of P-Tyr-RCML and P-Tyr-MBP is linear to $\sim 40 \%$. Samples were diluted for assay to maintain substrate dephosphorylation in the linear range.

PTPase activity was also measured with the autophosphorylated insulin receptor tyrosine kinase domain (P-Tyr-IRKD) as substrate. The 48-kD IRKD, purified from Sf9 cells after transfection with baculovirus expressing this domain, represents the intracellular domain of the insulin receptor beta subunit, lacking only eight amino acids adjacent to the transmembrane region (10). For use in an enzyme-linked immunosorbent assay (ELISA [11]), the IRKD was autophosphorylated in the presence of $20 \mathrm{mM}$ Hepes, $\mathrm{pH} 7.4,100 \mathrm{mM} \mathrm{NaCl}, 20 \mathrm{mM}$ p-nitrophenyl phosphate, $100 \mu \mathrm{M}$ vanadate, $2 \mathrm{mM} \mathrm{ATP,} 10 \mathrm{mM}$ $\mathrm{MgCl}_{2}, 5 \mathrm{mM} \mathrm{MnCl}$, and $30 \mu \mathrm{g} / \mathrm{ml}$ protamine for $30 \mathrm{~min}$ at $30^{\circ} \mathrm{C}$. The reaction was terminated by the addition of $20 \mathrm{mM}$ EDTA. The phosphodomain $(50 \mathrm{ng}$ ) was bound directly to microtiter plates in $50 \mathrm{mM}$ sodium carbonate/bicarbonate buffer, $\mathrm{pH}$ 9.6. Unbound sites were blocked with $2 \%$ BSA. Membrane extracts were preincubated at $30^{\circ} \mathrm{C}$ for $5 \mathrm{~min}$ in a solution containing $25 \mathrm{mM}$ Hepes, pH 7.2, 1 mM EDTA, $15 \mathrm{mM}$ 2-mercaptoethanol, and $1 \mathrm{mg} / \mathrm{ml} \mathrm{BSA}$. Reactions were initiated by addition of an aliquot $(50 \mu \mathrm{l})$ of the preincubation mixture to each well. After incubation for $30 \mathrm{~min}$ at $30^{\circ} \mathrm{C}$, the reaction was terminated by removal of the contents. The remaining unconverted phosphodomain was detected by incubation with $100 \mathrm{ng}$ of the mouse monoclonal anti-phosphotyrosine antibody PY69 (ICN Biomedicals Inc., Costa Mesa, CA) at $37^{\circ} \mathrm{C}$ for $1 \mathrm{~h}$, followed by horseradish peroxidase-conjugated rabbit IgG to mouse IgG (Dakopatts, London, England) at $37^{\circ} \mathrm{C}$ for $1 \mathrm{~h}$. The peroxidase was assayed colorimetrically using $3,3^{\prime}, 5,5^{\prime}$-tetramethylbenzidine. The ELISA is linear up to $60 \%$ and plateaus at $85 \%$ dephosphorylation of the P-Tyr-IRKD. This was established in experiments using multiple dilutions of four control rat liver particulate fractions. In these experiments, the relationship between phosphatase dilution and substrate dephosphorylation was linear to $60 \%$ with a correlation coefficient of 0.85 and a y intercept of $-4 \%$ dephosphorylation, which was not significantly different from the origin. In the presence of $100 \mu \mathrm{M}$ polyEY, dephosphorylation was inhibited by $90-100 \%$, indicating specificity for PTPases $(4,6)$.

A second assay using the soluble P-Tyr-IRKD was also used to verify the results obtained with immobilized IRKD in the ELISA. The 48-kD IRKD used in the soluble assay was autophosphorylated in vitro as follows. Approximately $5 \mu \mathrm{g}$ of IRKD protein $(5 \mu \mathrm{l})$ was preincubated for $15 \mathrm{~min}$ at $30^{\circ} \mathrm{C}$ in a final volume of $50 \mu$, containing $50 \mathrm{mM}$ HEPES, pH 7.6, $4 \mathrm{mM} \mathrm{MgCl} 2,0.3 \mathrm{mM}$ EDTA, $100 \mu \mathrm{g} / \mathrm{ml} \mathrm{BSA}$ and 20 $\mu \mathrm{g} / \mathrm{ml}$ protamine (Calbiochem Corp., La Jolla, CA). To this mixture was added $100 \mu \mathrm{M}\left[\gamma_{-}{ }^{32} \mathrm{P}\right] \mathrm{ATP}(100 \mu \mathrm{Ci})$, and incubation was continued for $1 \mathrm{~h}$ at $30^{\circ} \mathrm{C}$. Reaction was terminated by the addition of $5 \mu \mathrm{l}$ of $100 \mathrm{mM}$ EDTA. The P-Tyr-IRKD was precipitated in $10 \%$ trichloroacetic acid with $495 \mu \mathrm{g} \mathrm{BSA}$ added as a carrier. After $30 \mathrm{~min}$ on ice, the kinase mixture was centrifuged. The pellet was washed with $1 \mathrm{ml} 10 \%$ TCA and resuspended in $100 \mu \mathrm{l} 0.1 \mathrm{~N} \mathrm{NaOH}$. To this was added $400 \mu \mathrm{l}$ substrate dilution buffer (100 mM Hepes, pH 7.0, 1 mM EDTA, 15 $\mathrm{mM}$ 2-mercaptoethanol). The P-Tyr-IRKD was further diluted to 0.1 $\mu \mathrm{M}$ with substrate dilution buffer for use in the soluble PTPase assay (9). The P-Tyr-IRKD substrate was found to be a single phosphorylated band of $48 \mathrm{kD}$ on polyacrylamide gel electrophoresis. As in the case of the other PTPase assays, samples were diluted to give dephosphorylation within the linear range for this particular substrate.

Statistical analysis. Where noted, data are presented as mean \pm 1 SD. One-way analysis of variance (ANOVA) using the Scheffe $F$ test was used to assess for differences between groups. Linear regression was done by least squares using the GraphPad (San Diego, CA) Instat statistical package.

\section{Results}

Evidence for two PTPases differentially affected in diabetes. As noted above, there are two general classes of PTPases, the transmembrane receptor-like proteins that preferentially dephosphorylate P-Tyr-MBP, and the smaller PTPases $(\sim 50$ $\mathrm{kD})$ that display a lesser degree of substrate specificity. We demonstrated previously that alloxan diabetes did not affect the PTPase activity measured with acidic substrates (6). In contrast, rat liver membrane PTPase activity measured after $4 \mathrm{~d}$ of alloxan diabetes using P-Tyr-MBP as substrate showed an $86 \%$ increase in activity over normal animals (Fig. 1). Treatment with insulin resulted in a return towards normal levels. We also measured PTPase activity in rats fasted for $48 \mathrm{~h}$ before death to study the significance of the weight loss in diabetic animals. No significant changes in membrane PTPase activity with P-TyrMBP as substrate was seen in fasted animals. Neither cytoskeletal nor cytosolic P-Tyr-MBP PTPase activities were altered by 


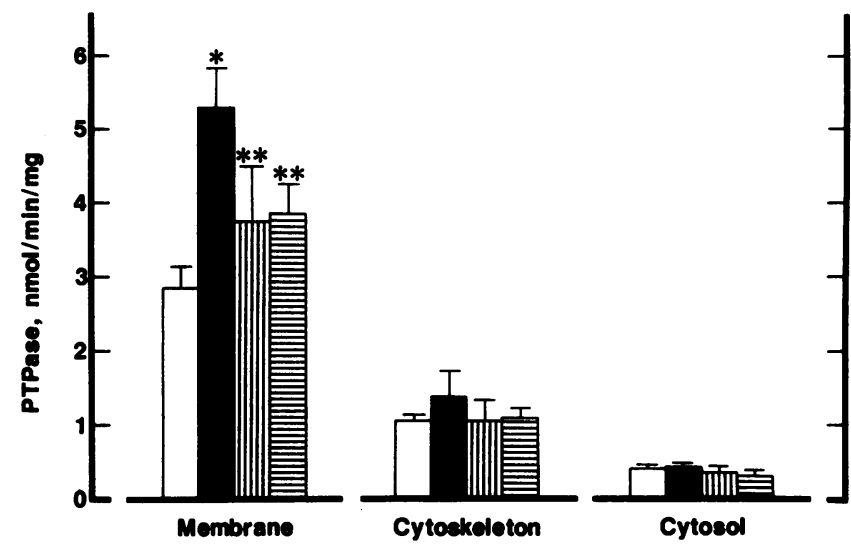

Figure 1. Effects of alloxan diabetes and fasting on rat liver PTPases measured with P-Tyr-MBP. Membrane, cytoskeleton, and cytosol fractions were prepared from control ( $n=5$, open bars), diabetic ( $n$ $=5$, solid bars), insulin-treated diabetic ( $n=6$, vertical cross-hatching), and fasted ( $n=5$, horizontal cross-hatching) rats. Fractions were assayed for PTPase activity using P-Tyr-MBP as substrate. Error bars represent 1 SD. A single asterisk denotes a significant difference from the corresponding control group. Two asterisks denote a significant difference from the corresponding diabetic group.

alloxan diabetes or fasting, showing that the effect was specific for a particular PTPase activity in one subcellular fraction.

The specific PTPase activity of the membrane fraction from normal rats was 2.7-fold higher than the cytoskeletal fraction with P-Tyr-MBP as substrate (Fig. 1). In contrast, membrane and cytoskeleton specific activities measured on the same fractions with P-Tyr-RCML were nearly identical (data not shown). The latter finding was in agreement with our previous studies (6). Cytosolic PTPase specific activity with P-TyrMBP was approximately seven-fold lower than membrane specific activity in normal animals (Fig. 1). Our results are consistent with the conclusion that activity measured with P-Tyr-MBP resides in membrane-associated PTPases.

As noted above, fractions were also analyzed for PTPase activity using P-Tyr-RCML. Although a modest increase in PTPase activity in the membrane fraction from diabetic rats $(1.2 \pm 0.2 \mathrm{nmol} / \mathrm{min}$ per $\mathrm{mg}$ protein [mean $\pm \mathrm{SD}$ ] vs. $0.8 \pm 0.1$ in normal animals) was detected, this increase in PTPase activity persisted after insulin treatment $(1.1 \pm 0.1 \mathrm{nmol} / \mathrm{min}$ per $\mathrm{mg}$ protein). No significant change in the membrane fraction from fasted rats $(1.0 \pm 0.03 \mathrm{nmol} / \mathrm{min}$ per $\mathrm{mg}$ protein) was detected. The cytoskeletal and cytosolic fractions showed no change in PTPase activity, as found previously (6).

HPLC gel filtration chromatography of rat hepatic membrane-associated PTPases. We separated membrane PTPases by size using HPLC gel filtration chromatography. Liver membranes from a normal rat were extracted with $1 \%$ Triton X-100. The extracted membrane proteins were separated using a column (SW-300; Waters Chromatography Division; Milford, MA) equilibrated in $50 \mathrm{mM}$ Hepes, $\mathrm{pH} 7.0,150 \mathrm{mM} \mathrm{NaCl}, 15$ $\mathrm{mM}$ 2-mercaptoethanol, and $0.1 \%$ Triton X-100. Fractions $(0.3 \mathrm{ml})$ were collected and assayed for PTPase activity using both P-Tyr-RCML and P-Tyr-MBP.

PTPase activity measured with P-Tyr-RCML was present in two distinct peaks, $\sim 450$ and $44 \mathrm{kD}$ (Fig. 2). In contrast, PTPase activity with P-Tyr-MBP as substrate was present only in a higher molecular weight peak. The ratio of activity with P-Tyr-MBP to P-Tyr-RCML in this peak was $\sim 6: 1$. Analysis of several paired control and alloxan diabetic membrane extracts revealed no consistent qualitative changes in the distribution of activities between high and low molecular weight peaks.

Characterization of the high molecular weight rat liver PTPase. The finding that the PTPase with preferential activity towards P-Tyr-MBP eluted during gel filtration chromatography at $\sim 450 \mathrm{kD}$ is similar to findings with CD45 (180-200 $\mathrm{kD}$ ) which yielded a peak of P-Tyr-MBP PTPase activity at greater than $650 \mathrm{kD}$ during gel filtration chromatography (5). Although CD45 is not expressed in cells other than leukocytes, several related PTPases are potential candidates for a hepatic, CD45-like PTPase (3). We therefore studied the high molecular weight PTPase partially purified from liver membranes by poly-L-lysine agarose affinity chromatography (see below and reference 7) to detect characteristics similar to those described for CD45.

Activity of the high molecular weight liver enzyme with P-Tyr-RCML as substrate was increased by the addition of myelin basic protein (Fig. $3 A$ ). The same experiment performed on the low molecular weight PTPases exhibited a decrease in PTPase activity of greater than $60 \%$ (not shown). Activity of the high molecular weight enzyme with P-Tyr-RCML as substrate was also increased by treatment with trypsin, while the activity using P-Tyr-MBP as substrate was unaffected (Fig. 3 $B$ ). The findings in Fig. 3 are similar to those from similar experiments by Tonks et al. (5) using CD45.

Dephosphorylation of the 48-kD insulin receptor tyrosine kinase domain. Our earlier studies (6) on alloxan diabetes in the rat had indicated a slight decrease rather than an increase in insulin receptor dephosphorylation. Given the present results that PTPase activity with P-Tyr-MBP increased in alloxan diabetes, we sought to determine whether the PTPase measured with P-Tyr-MBP corresponded to the enzyme responsible for insulin receptor dephosphorylation. The ELISA (see Methods) used to measure the percent dephosphorylation of autophos-

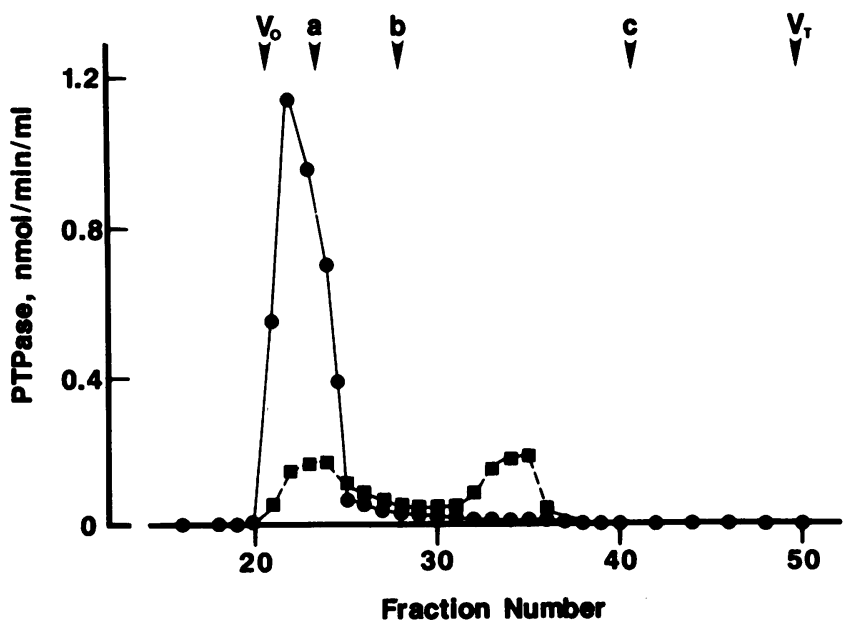

Figure 2. Separation of rat liver membrane PTPases by HPLC gel filtration chromatography. An extract of rat liver membranes was applied to a 15-ml SW-300 HPLC gel filtration column equilibrated in $50 \mathrm{mM}$ Hepes, pH 7.0, $150 \mathrm{mM} \mathrm{NaCl}, 15 \mathrm{mM}$ 2-mercaptoethanol, and $0.1 \%$ Triton X-100. Fractions were assayed for PTPase activity using P-Tyr-MBP (๑) and P-Tyr-RCML ( () as substrate. Symbols at the top of the figure denote the calibration of the column: $V_{o}$, void volume; $a$, ferritin (440 kD); $b$, aldolase (158 kD); $c$, ribonuclease A $(13.7 \mathrm{kD}) ; V_{T}$, total column volume. 


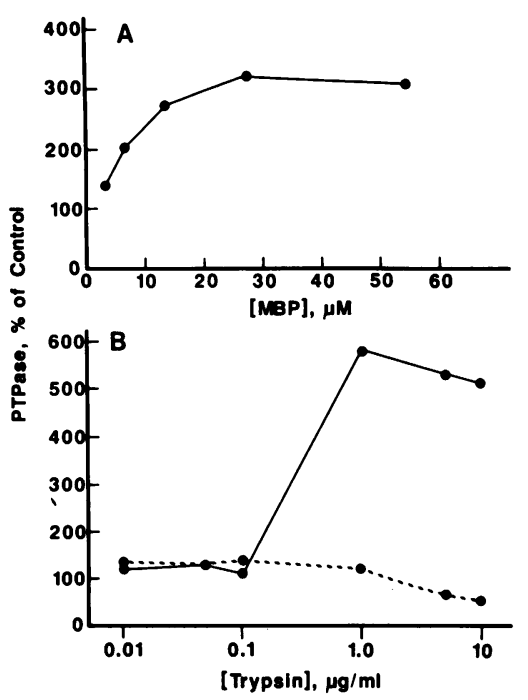

Figure 3. Activation of the high molecular weight rat liver membrane PTPase by myelin basic protein or trypsin. (A) Activity of partially purified $450-\mathrm{kD}$ rat liver membrane PTPase was measured with P-TyrRCML as substrate in the presence of varying concentrations of myelin basic protein. $(B)$ The same PTPase preparation used for the experiment shown in $A$ was incubated with varying concentrations of trypsin for $10 \mathrm{~min}$ at $30^{\circ} \mathrm{C}$. Proteolysis was terminated by the addi-

tion of an equal amount (weight/weight) of lima bean trypsin inhibitor. PTPase activity then was measured using P-Tyr-RCML (solid line) or P-Tyr-MBP (dashed line) as substrate.

phorylated P-Tyr-IRKD was applied to Triton X-100 extracts of rat liver membranes from normal, alloxan diabetic, treated diabetic, and fasted animals (Fig. 4, left). PTPase activity also was measured with both P-Tyr-RCML and P-Tyr-MBP in these extracts.

There was no significant difference in P-Tyr-IRKD dephosphorylation between any of the experimental groups. Comparison of the percent IRKD dephosphorylation versus PTPase activity with P-Tyr-RCML (Fig. 4, center) yielded a correlation coefficient of $0.76(P<0.01)$. In contrast, IRKD dephosphorylation and PTPase activity with P-Tyr-MBP (Fig. 4, right) were not correlated $(r<0.01)$. These data were interpreted to suggest that the PTPases measured with P-Tyr-RCML account for the preponderance of insulin receptor dephosphorylation.

To study this further, rat liver membrane PTPases separated by poly-L-lysine agarose affinity chromatography were examined for their relative activities with the two artificial substrates (P-Tyr-RCML and P-Tyr-MBP) and the P-Tyr-IRKD. To confirm the validity of results using immobilized P-TyrIRKD in an ELISA system, activity on poly-L-lysine fractions was measured using soluble P-Tyr-IRKD in our usual PTPase assay. Poly-L-lysine chromatography (Fig. 5) resulted in a separation of activity with P-Tyr-RCML from activity with P-TyrMBP. Assays on individual fractions showed that soluble PTyr-IRKD dephosphorylation coincided with P-Tyr-RCML dephosphorylation. Linear regression analysis of PTPase activities with P-Tyr-RCML and P-Tyr-IRKD showed a highly significant correlation $(r=0.88, P<0.0001)$. There was no significant correlation between PTPase activities with P-Tyr-MBP and P-Tyr-IRKD $(r=0.37)$, or P-Tyr-MBP and P-Tyr-RCML $(r=0.41)$.

In our previous studies (7) we used inhibition of PTPase activity by poly(glutamic acid:tyrosine [4:1]) (polyEY) to distinguish between membrane PTPases I, II, and III separated during poly-L-lysine agarose affinity chromatography. To further ascertain whether PTPase activity with P-Tyr-IRKD paralleled activity with P-Tyr-RCML or P-Tyr-MBP, polyEY $(0-20 \mu \mathrm{M})$ was added to a membrane extract before determination of PTPase activity using all three substrates (Fig. 6). Dephosphor- ylation of P-Tyr-IRKD by the mixture of PTPases in an unfractionated membrane extract was inhibited by polyEY in a manner identical to that seen using P-Tyr-RCML as substrate. In contrast, PTPase activity with P-Tyr-MBP showed a biphasic effect of polyEY. Activity was stimulated at concentrations below $10 \mu \mathrm{M}$ and inhibited at $20 \mu \mathrm{M}$. The results shown in Fig. 6 were consistent with those seen using membrane PTPases I, II, and III in our prior study (7). They are, therefore, interpreted as further indication that hepatic membrane PTPases that show preferential activity towards P-Tyr-RCML largely account for P-Tyr-IRKD dephosphorylation.

\section{Discussion}

In the present study, rats made diabetic with alloxan exhibited a substantial increase in rat liver membrane PTPase activity measured with P-Tyr-MBP. In contrast to PTPase activity with P-Tyr-RCML as substrate ([6] and the present report), our data show that PTPase specific activity with P-Tyr-MBP is highest in the membrane fraction. Using molecular cloning techniques, Streuli et al. (12) have described transmembrane PTPases related to CD45 which they termed LAR (leukocyte antigen related). A preliminary report describes the detection of mRNA in rat liver using the cDNA for LAR (13). We have found that the high molecular weight P-Tyr-MBP PTPase from rat liver membranes, partially purified by poly-L-lysine agarose affinity chromatography (7), has characteristics in common with CD45. It was stimulated by the polycation, myelin basic protein, whereas the low molecular weight PTPases were inhibited. Limited proteolysis with trypsin revealed a sixfold increase in activity, while the low molecular weight PTPases showed no change. The enzyme in rat liver that we are measuring is unlikely to be CD45, expression of which has only been found in leukocytes (3). Direct comparison with data using LAR is not possible because such studies have not been published. Nonetheless, our data support the hypothesis that the
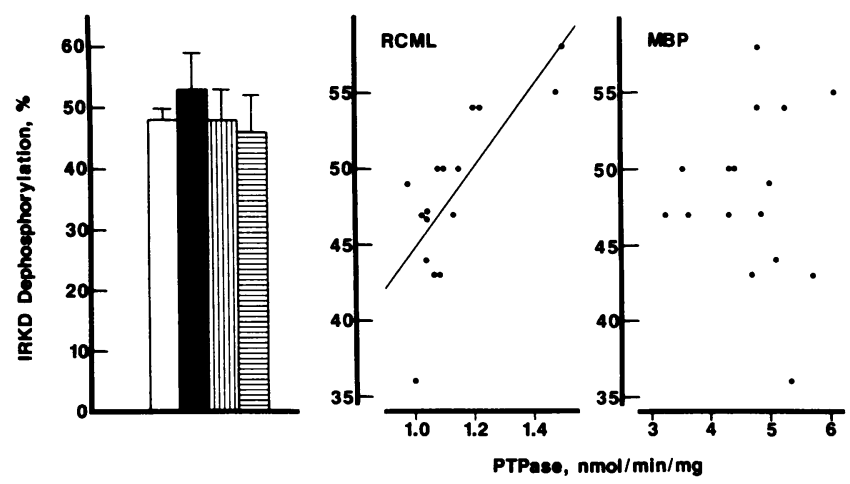

Figure 4. Dephosphorylation of the $48-\mathrm{kD}$ insulin receptor tyrosine kinase domain (IRKD) by rat liver membrane PTPase. (Left) Membrane fractions prepared from control $(n=4$, open bar), diabetic ( $n$ $=4$, solid bar), insulin-treated diabetic ( $n=4$, vertical cross-hatching) and fasted ( $n=4$, horizontal cross-hatching) rats were used to dephosphorylate the 48-kD IRKD in an ELISA as described in Methods. Rat liver membrane extracts were used at identical protein concentrations, $0.36 \mathrm{mg} / \mathrm{ml}$ in the assay. Error bars represent $1 \mathrm{SD}$. (Center) Individual data points for each animal from the four groups; percent dephosphorylation of P-Tyr-IRKD versus P-Tyr-RCML PTPase activity ( $\mathrm{nmol} / \mathrm{min}$ per $\mathrm{mg}$ protein) in each sample $(r=0.76)$. (Right) Percent dephosphorylation of P-Tyr-IRKD versus P-Tyr-MBP PTPase activity ( $\mathrm{nmol} / \mathrm{min}$ per $\mathrm{mg}$ protein) in each sample $(r$ $=-0.009)$. 

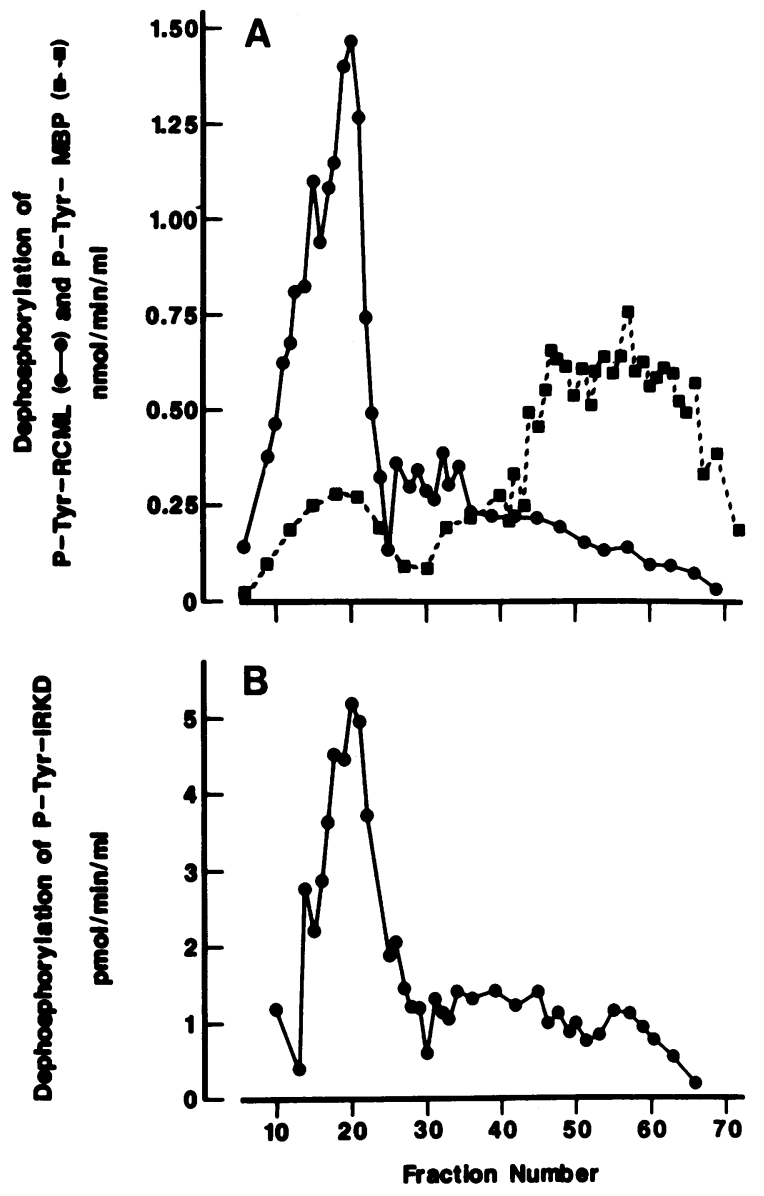

Figure 5. Dephosphorylation of P-Tyr-RCML, P-Tyr-MBP, and PTyr-IRKD by PTPases fractionated using poly-L-lysine agarose affinity chromatography. Proteins extracted from rat liver membranes using $1 \%$ Triton X-100 were adsorbed to a $20-\mathrm{ml}$ column of poly-Llysine agarose equilibrated in $20 \mathrm{mM}$ Hepes, pH 7.4, 2 mM EDTA, $25 \mathrm{mM} \mathrm{NaCl}, 1 \mathrm{mM}$ benzamidine, $0.002 \% \mathrm{PMSF}$, and $0.4 \%$ 2-mercaptoethanol. PTPases were eluted with a linear gradient from 25 $\mathrm{mM}$ to $1 \mathrm{M} \mathrm{NaCl}(400 \mathrm{ml}$, total elution volume). PTPase activity of individual fractions $(4 \mathrm{ml})$ was measured using P-Tyr-RCML $(\bullet, A)$, P-Tyr-MBP (n, $A$ ), and P-Tyr-IRKD $(B)$ as substrates. Activities with all three substrates were determined in the same soluble dephosphorylation assay.

activity of LAR-like PTPase(s) in rat liver is increased in alloxan diabetes.

We sought to determine if an increase in LAR-like PTPase(s) might increase insulin receptor dephosphorylation. We have shown that membrane extracts from normal and alloxan diabetic rats contained PTPase activity that dephosphorylated partially purified hepatic insulin and epidermal growth factor (EGF) receptors (6). We showed further that alloxan diabetes produced a subtle decrease in insulin receptor dephosphorylation (6). In a subsequent publication (7), we indicated that the rat liver membrane PTPase III partially purified by poly-L-lysine agarose chromatography could dephosphorylate the P-Tyr-insulin receptor beta subunit. However, these experiments used insulin receptors prepared from rat liver membranes and phosphorylated in vitro. Substrate (receptor) concentrations were very low and probably comparable to PTPase concentrations. Therefore, these experiments may not provide an indication of the relative activities of the three PTPases with the autophosphorylated insulin receptor beta subunit. To further characterize the insulin receptor as a substrate for PTPases, the dephosphorylation of a 48-kD, autophosphorylated P-Tyr-IRKD was monitored. This approach offered the advantage of higher substrate concentrations. Using this approach, we found that alloxan diabetes and fasting caused no change in the extent of P-Tyr-IRKD dephosphorylation by liver membrane detergent extracts. Correlation between P-TyrIRKD and artificial substrate dephosphorylation by these extracts indicated that P-Tyr-RCML and insulin receptors probably are dephosphorylated by the same PTPases. This led to the conclusion that the observed increase in PTPase activity with P-Tyr-MBP seen in alloxan diabetes may be unassociated with a change in insulin receptor dephosphorylation.

Several other observations support this conclusion. Fractionation of liver membrane PTPases by poly-L-lysine agarose chromatography showed a strong correlation between dephosphorylation of P-Tyr-RCML and P-Tyr-IRKD. In contrast, PTPase activity with P-Tyr-MBP, largely confined to the activity we have designated previously as PTPase III (7), did not coincide with P-Tyr-IRKD dephosphorylation. Similarly, inhibition of membrane extract PTPase activities by polyEY showed identical behavior of P-Tyr-RCML and P-Tyr-IRKD as substrates. The experiments shown in Figs. 5 and 6 , using soluble P-Tyr-IRKD as substrate, were consistent with those in Fig. 4 which used immobilized P-Tyr-IRKD in an ELISA system. These results, taken together, indicate that P-Tyr-RCML PTPases in rat liver membranes account for the greater part of P-Tyr-IRKD phosphatase activity.

Our original hypothesis was that an increase in PTPase activity might contribute to insulin resistance. An impaired response to insulin is associated with insulin-deficient (type 1) diabetes mellitus, despite an increased number of insulin receptors measured by hormone binding $(14,15)$. The defect manifests as decreased insulin stimulation of glucose transport, glucose oxidation, and lipogenesis in skeletal muscle (16), adipose tissue (17-19), and liver $(20,21)$. The specific events leading to these metabolic changes in the cell have not been identified. However, a broad spectrum of approaches yielded results supporting the role of tyrosine phosphorylation in the insulin

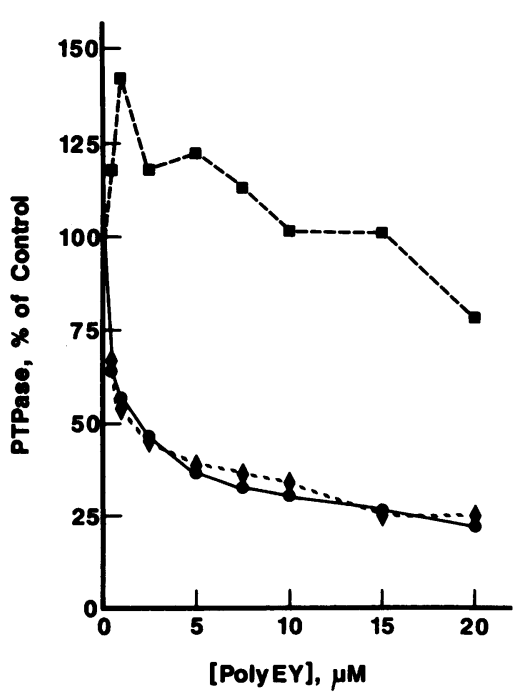

Figure 6. Effect of poly(glutamic acid:tyrosine [4:1]) on liver membrane extract PTPase activities. A control rat liver membrane detergent extract was preincubated with varying concentrations of polyEY for $5 \mathrm{~min}$ at $30^{\circ} \mathrm{C}$. PTPase activity was then determined using P-Tyr-RCML (•), P-Tyr-MBP ( $(\bullet)$ and PTyr-IRKD ( $\bullet$ as substrates. Activities with all three substrates were determined in the same soluble dephosphorylation assay. Results are expressed as percentage of control (no polyEY). 
signaling process. Type 1 diabetes has been associated with a decrease in receptor autophosphorylation as well as receptor kinase activity (reviewed by Haring and Obermaier-Kusser [22]). Blondel et al. (23) have reported that oral administration of vanadate (a PTPase inhibitor) to 10-wk-old female rats with non-insulin-dependent diabetes causes a corrective insulinlike effect on impaired insulin action in both the liver and peripheral tissues. We hypothesized that the impaired metabolic response to insulin in diabetes might involve an increase in PTPase activity for the insulin receptor and/or its substrates. Our data indicate that in the specific case of alloxan diabetes in the rat, there is indeed a change in PTPase activity as measured with P-Tyr-MBP. However, this appears to be unaccompanied by changes in insulin receptor PTPases. Therefore, a possible role for the P-Tyr-MBP PTPase in modulating insulin action will require uncovering the physiologic substrates for the insulin receptor tyrosine kinase, as well as their corresponding phosphatases.

\section{Acknowledgments}

We thank Patricia Carter for her contribution to these studies. We also appreciate the secretarial assistance of Donna Berger.

This work was supported by National Institutes of Health grants HD-24455 (to Philip A. Gruppuso), GM-35266 (to David L. Brautigan) and DK-40511 (to Leland Ellis).

\section{References} 458.

2. Cicirelli, M. F., N. K. Tonks, C. D. Diltz, J. W. Weiel, E. H. Fischer, and E. G. Krebs. 1990. Microinjection of a protein-tyrosine-phosphatase inhibits insulin action in Xenopus oocytes. Proc. Natl. Acad. Sci. USA. 87:5514-5518.

3. Fischer, E. H., H. Charbonneau, and N. K. Tonks. 1991. Protein tyrosine phosphatases: a diverse family of intracellular and transmembrane enzymes. Science (Wash. DC). 253:401-406, 1991.

4. Tonks, N. K., C. D. Diltz, and E. H. Fisher. 1988. Characterization of the major protein-tyrosine-phosphatases of human placenta. J. Biol. Chem. 263:6731-6737.

5. Tonks, N. K., C. D. Diltz, and E. H. Fisher. 1990. CD45, an integral membrane protein tyrosine phosphatase: characterization of enzyme activity. $J$. Biol. Chem. 265:10674-10680.

6. Gruppuso, P. A., J. M. Boylan, B. I. Posner, R. Faure, and D. L. Brautigan. 1990. Hepatic protein phosphotyrosine phosphatase. Dephosphorylation of insu- lin and epidermal growth factor receptors in normal and alloxan diabetic rats. $J$. Clin. Invest. 85:1754-1760.

7. Gruppuso, P. A., J. M. Boylan, B. L. Smiley, R. L. Fallon, and D. L. Brautigan. 1991. Hepatic protein tyrosine phosphatases in the rat. Biochem. J. 274:361-367.

8. Williams, L. W., P. M. Tremble, M. F. Lavin, and M. S. Sunday. 1984. Platelet-derived growth factor receptors form a high affinity state in membrane preparations: kinetics and affinity cross-linking studies. J. Biol. Chem. 259:52875294.

9. Shriner, C. L., and D. L. Brautigan. 1984. Cytosolic protein phosphotyrosine phosphatases from rabbit kidney. J. Biol. Chem. 259:11383-11390.

10. Ellis, L., A. Levitan, M. H. Cobb, and P. Ramos. 1988. Efficient expression in insect cells of a soluble, active human insulin receptor protein-tyrosine kinase domain by use of a baculovirus vector. J. Virol. 62:1634-1639.

11. Madden, J. A., M. I. Bird, Y. Man, T. Raven, and D. D. Myles. 1991. Two nonradioactive assays for phosphotyrosine phosphatases with activity toward the insulin receptor. Anal. Biochem. 199:210-215.

12. Streuli, M., N. X. Krueger, L. R. Hall, S. F. Schlossman, and H. Saito. 1988. A new member of the immunoglobulin superfamily that has a cytoplasmic region homologous to the leukocyte common antigen. J. Exp. Med. 168:15231530.

13. Zhang, W. R., C. R. Kahn, and B. J. Goldstein. 1990. Identification of a family of rat liver protein-tyrosine phosphatases by cDNA cloning. In Program and Abstracts, The Endocrine Society, 72nd Annual Meeting. 72a. (Abstr.)

14. Kadowaki, T., M. Kasuga, Y. Akanuma, O. Ezaki, and F. Takaku. 1984. Decreased autophosphorylation of the insulin receptor-kinase in streptozotocindiabetic rats. J. Biol. Chem. 259:14208-14216.

15. Burant, C. F., M. K. Treutelaar, and M. G. Buse. 1986. Diabetes-induced functional and structural changes in insulin receptors from rat skeletal muscle. $J$. Clin. Invest. 77:260-270.

16. Kipnis, D. M., and C. F. Cori. 1959. Studies of tissue permeability. V. The penetration and phosphorylation of 2-deoxyglucose in the rat diaphragm. J. Biol. Chem. 234:171-177.

17. Kasuga, M., Y. Akanuma, Y. Iwamoto, and K. Kosaka. 1978. Insulin binding and glucose metabolism in adipocytes of streptozotocin-diabetic rats. Am. J. Physiol. 235:E175-E182.

18. Kobayashi, M., and J. M. Olefsky. 1979. Effects of streptozotocin-induced diabetes on insulin binding, glucose transport, and intracellular glucose metabolism in isolated rat adipocytes. Diabetes. 18:87-95.

19. Karnieli, E., P. J. Hissin, I. A. Simpson, L. B. Salans, and S. W. Cushman. 1981. A possible mechanism of insulin resistance in the rat adipose cell in streptozotocin-induced diabetes mellitus. J. Clin. Invest. 68:811-814.

20. Haft, D. E. 1968. Studies of the metabolism of isolated livers of normal and alloxan-diabetic rats perfused with insulin. Diabetes. 17:244-250

21. Marchand, Y.-L., E. G. Loten, F. Assimacopoulos-Jeannet, M.-E. Forgue, P. Freychet, and B. Jeanrenaud. 1976. Effect of fasting and streptozotocin in the obese-hyperglycemic (ob/ob) mouse: apparent lack of a direct relationship between insulin binding and insulin effects. Diabetes. 26:582-590.

22. Haring, H., and B. Obermaier-Kusser. 1989. Insulin receptor kinase defects in insulin-resistant tissues and their role in the pathogenesis of NIDDM. Diabetes Metab. Rev. 5:431-441.

23. Blondel, O., J. Simon, B. Chevalier, and B. Portha. 1990. Impaired insulin action but normal insulin receptor activity in diabetic rat liver: effect of vanadate. Am. J. Physiol. 258:E459-E467. 